\title{
Robust Backstepping Controller for Inner and Outer Loops Control of a Small-Scale Helicopter
}

\author{
Tushar K. Roy \\ Department of ETE, RUET, Bangladesh \\ *Corresponding Author: roy_kanti@yahoo.com
}

Copyright $@ 2014$ Horizon Research Publishing All rights reserved.

\begin{abstract}
This paper presents an investigation into the design of a flight control system, using a robust backstepping control structure, designed using the two-time scale control procedure of the dynamic of a small-size autonomous helicopter in hover. The two-time scale controller takes advantage of the 'decoupling' of the translational and rotational dynamics of the rigid body, resulting in a two-level hierarchical control scheme. The inner loop controller (attitude control) tracks the attitude commands generated by the outer loop controller and sets the main rotor thrust vector, while the outer loop controller (position control) tracks the reference position. The controller is designed using the backstepping method based on Lyapunov function. Lyapunov function is used to show the robustness of the proposed control method under the consideration of wind gusts. Simulation results are finally given showing the effectiveness of the proposed control and its ability to cope with external wind gusts on the plant model.
\end{abstract}

Keywords Helicopter Dynamics, Lyapunov Stability, Robust Backstepping Control, Uncertainty

\section{Introduction}

Recently, the core of aerospace industries has moved to UAV (Unmanned Aerial Vehicle), which is better than manned aircraft in some respects such as flight stability for highly dangerous works, physical Limits caused by long-time flight or sudden maneuver, enormous time and expenditure cost for pilot training. Among the variety of Unmanned Air Vehicles (UAVs), unmanned autonomous helicopters (UAH) constitute one of the most versatile and agile platforms. A helicopter can operate in different flight modes, such as vertical take-off/landing, hovering, longitudinal/lateral flight, and bank to turn which gives them the advantage of effective observation from various positions. Among these abilities hovering and vertical take-off is necessarily needed. Unmanned autonomous helicopter control system should make these performances achieved by improving the tracking performance and disturbance rejection capability in different weather conditions. Unmanned autonomous helicopter control system should make these performances achieved by improving the tracking performance and disturbance rejection capability in different weather conditions. Therefore, robustness is one of the critical issues which must be considered in the control system design for small unmanned autonomous helicopter, especially those covering large flight envelope. One major problem rarely addressed by researchers to date is that of a wind disturbance. To cope with such a problem, researchers have considered different approaches such as robust feedback linearization, robust nonlinear $\mathrm{H} \infty$ controller $[1,2]$, neural network [3, 4], adaptive backstepping [5] etc. This paper presents a robust backstepping controller design to stabilize the inner loop and the outer loop of a small scale helicopter in the presence of external wind gusts. The control of a hover flight in a gusty environment is always a challenging control problem due to the inherent nonlinearity, dynamical instability and unsteady operating conditions. The position control of a small scale unmanned autonomous helicopter under the vertical and horizontal wind gusts using the feedback method is addressed in [6], wherein the wind disturbance is assumed to be the sum of a fixed number of sinusoids with unknown amplitudes, frequencies and phases. In [7], the authors present a robust backstepping technique of autonomous scale helicopter subject to parameters uncertainties and uniform time varying three-dimensional wind gusts. With the assistance of an unknown input observer technique (UIO), the controller is reported to be able to handle the effect of these uncertainties on an autonomous helicopter. A robust $\mathrm{H}_{\infty}$ controller for a hover flight is addressed in [8], in which the effects of external wind gusts are considered within the nonlinear helicopter model to cause random fluctuations in body velocities. Position control of a small helicopter using backstepping controller including disturbance observer and flapping dynamics is presented in [9]. A robust backstepping controller to control the lateral and longitudinal position for hover flight mode of an unmanned autonomous helicopter (UAH) in a gusty environment is considered in [10], in which the author considers the linear model of small helicopter. 
The main contribution of this article consists in proposing a robust nonlinear control of an autonomous scale helicopter subject to disturbances as wind gusts. This paper presents a two layer control structure consisting of an inner-loop and an outer-loop flight controller for an unmanned autonomous helicopter. The outer-loop controller is responsible for the position control along with the altitude, whereas the inner-loop controller is responsible for the attitude control of a small helicopter. To design the controller, external disturbances are taken into account within the system model. Lyapunov technique is used to show the robustness of the proposed method under the consideration of wind gusts. In order to validate the feasibility of the proposed control method, the computer simulation results are shown.

The rest of this paper is organized as follows. Section II, briefly introduces the mathematical model used. Section III, presents the gusts model. Section IV, presents a hierarchical controller, including an outer-loop and inner-loop controller, is designed for both subsystems. Section V, discusses simulation results. Finally, the paper concludes in Section VI.

\section{Rigid Body Dynamics}

This section displays the 6-DOF dynamic model of a miniature helicopter. The nonlinear rigid body dynamics of the UAV helicopter in terms of translational and rotational dynamics can be expressed by the following Newton-Euler equations:

$$
\begin{aligned}
& \dot{\zeta}=V \\
& m \dot{V}=R f_{b}+m g e_{3} \\
& \dot{\eta}=\pi \omega \\
& I \dot{\omega}=\omega \times I \omega+M
\end{aligned}
$$

where, $m$ is the total mass of the UAV helicopter, $I$ is the inertia matrix, $\omega=[p, q, r]^{T}$ represents the helicopter angular rate vector. The gravitational force $m g e_{3}$ is explicitly included where, $e_{3}$ is a unit vector with one in the third place. The Euler angle vector $\eta=[\phi, \theta, \psi]^{T}$ is defined in the roll-pitch-yaw sequence. Therefore, the helicopter's rotation matrix $R$ in terms of Euler angles is given by [5]

$$
R=\left[\begin{array}{ccc}
c_{\theta} c_{\psi} & s_{\phi} s_{\theta} c_{\psi}-c_{\phi} s_{\psi} & c_{\phi} s_{\theta} c_{\psi}+s_{\phi} s_{\psi} \\
c_{\theta} s_{\psi} & s_{\phi} s_{\theta} s_{\psi}+c_{\phi} c_{\psi} & c_{\phi} s_{\theta} s_{\psi}-s_{\phi} c_{\psi} \\
-s_{\theta} & s_{\phi} c_{\theta} & c_{\phi} c_{\theta}
\end{array}\right]
$$

Where $c(),. s($.$) are abbreviations for \cos (),. \sin ($.$) . A$ conventional single main rotor helicopter has four independent control inputs. The control inputs are defined as

$u_{c}=\left[T_{m}, T_{t}, a_{1}, b_{1}\right]^{T}$. The components $T_{m}$ and $T_{t}$ are the magnitude of the generated thrusts, respectively by main and tail rotor. In helicopters and RUAVs, the dominant response is due to the main and tail rotor. $a_{l}$ and $b_{l}$ are longitudinal and lateral flapping angles to the tip path plane of the main rotor with respect to the shaft, respectively. For the purpose of simplification, most of the researchers design the controllers by only considering the main and tail rotor and ignore the horizontal stabilizer, vertical stabilizer and fuselage stabilizer. The force generated by main rotor $f_{b}$ along the $\left(x_{b}\right.$, $y_{b}, z_{b}$ ) direction in body coordinates can be written as:

$$
f_{b}=\left[X_{m}, Y_{m}, Z_{m}\right]^{T}
$$

The following are the force components due to main rotor thrusts

$$
\begin{aligned}
& X_{m}=-T_{m} \sin \left(a_{1}\right) \\
& Y_{m}=T_{m} \sin \left(b_{1}\right) \\
& Z_{m}=-T_{m}
\end{aligned}
$$

The tail rotor generates force in $y_{b}$ direction only. Therefore, the force generated by the tail rotor is given by

$$
\left[X_{t}, Y_{t}, Z_{t}\right]^{T}=[0,-1,0]^{T} T_{t}
$$

Therefore, by including the helicopter's weight the complete force vector will be

$$
f_{b}=\left[\begin{array}{c}
-T_{m} \sin \left(a_{1}\right) \\
T_{m} \sin \left(b_{1}\right)-T_{t} \\
-T_{m}
\end{array}\right]+R^{T}\left[\begin{array}{c}
0 \\
0 \\
m g
\end{array}\right]
$$

And net moments acting on the helicopter are described as

$$
M=\left[\begin{array}{c}
L \\
R \\
N
\end{array}\right]=\left[\begin{array}{c}
L_{b} b_{1}+Y_{m} M_{z}+T_{t} T_{z} \\
M_{a} a_{1}+X_{m} M_{z} \\
M_{Q}+Y_{m} M_{x}+T_{t} T_{x}
\end{array}\right]
$$

where $L_{b}, M_{a}$ are the effective flap-stiffness constants. The main rotor lengths $M_{x}, M_{z}$ and tail rotor lengths $T_{x}, T_{z}$ are with respect to the center of mass of the body. There exit a simple relation [5] between $M_{Q}$ drag vector and main rotor thrust $T_{m}$. The relation is given as follows:

$$
M_{Q}=C_{m} T_{m}^{1.5}+D_{m}
$$

where $C_{m}=\frac{1}{\Omega \sqrt{2 \rho A}}$ is the coefficient expressing the relation between main rotor thrust and drag; $D_{m}$ is the initial drag of the main rotor when the blade pitch is zero. The aerodynamic forces and moments due to empennage and fuselage are negligible in hovering and low-velocity flight thus they are neglected for the controller design.

\section{Gust Model}

The purpose of including wind gusts into the simulation model is to ensure that the design controller can cope with a real-world environment where wind effects can be a 
significant challenge to station keeping. For analysis of RUAV, wind gusts can be treated as either random (spectral turbulence) or discrete. For random gusts, typical spectral models include the Von Karman and Dryden turbulence models. The Von Karman model has been widely considered the more "realistic" model when it comes to defining turbulence spectra. However, due to the computational complexity of the Von Karman model, the Dryden model is typically used in aerospace vehicle analyses. There are many sources for wind models based upon empirical data that consist of passing band limited white noise through appropriate forming filters. The turbulence models are scaled with respect to RUAV altitude, velocity, wing span. The filters used to generate the Dryden spectral model of atmospheric disturbance [11] are given by:

$$
\begin{gathered}
H_{u}(s)=\sigma_{u} \sqrt{\frac{2 L_{u}}{\pi U}} \frac{1}{1+\frac{L_{u}}{U} s} \\
H_{v}(s)=\sigma_{v} \sqrt{\frac{L_{v}}{\pi U}} \frac{1+\frac{\sqrt{3} L_{v}}{U} s}{\left(1+\frac{L_{v}}{U} s\right)^{2}} \\
H_{w}(s)=\sigma_{v} \sqrt{\frac{L_{w}}{\pi U}} \frac{1+\frac{\sqrt{3} L_{w}}{U} s}{\left(1+\frac{L_{w}}{U} s\right)^{2}}
\end{gathered}
$$

where, $\mathrm{U}$ is the true speed of a RUAV, $\sigma_{u}$, and $\sigma_{v}$ are the root mean square intensities of the turbulence and $L_{u}, L_{v}$ and $L_{w}$ are the turbulence scale lengths that describe the behavior of the wind gusts. In this work, the scale of turbulence, $L_{u}$, and $L_{v}$ are assigned constant values of $L_{u}=L_{v}=722.5 \mathrm{~m}$ and $L_{w}=h$. And for the low altitude region (altitude $<1000 \mathrm{ft}$ ) the $\sigma_{u}, \sigma_{v}$, and $\sigma_{w}$ turbulence intensities are given by

$$
\begin{aligned}
\sigma_{w} & =0.1 W_{20} \\
\frac{\sigma_{u}}{\sigma_{w}}=\frac{\sigma_{v}}{\sigma_{w}} & =\frac{1}{(0.177+0.000823 h)^{0.4}}
\end{aligned}
$$

where, $W_{20}$ is the wind speed at $20 \mathrm{ft}(6 \mathrm{~m})$ above the ground and can be approximated by $U$ and altitude is described by $h$. In this paper, we consider a typical level of wind speed is 3 $\mathrm{m} / \mathrm{s}$ and altitude is $-2 \mathrm{~m}$.

\section{Backstepping Controller Design}

In this section, a hierarchical inner-outer loop approach is used to design the controller for a small helicopter. In this control structure, the RUAV system is stabilized in the inner-loop and then it is driven to track a desired position along with the altitude in the outer-loop.

\section{A. Outer-loop controller design}

In this section, a robust backstepping controller will be designed to keep the position of a small helicopter in a desired way. Theoretically speaking, our aim is to design a control law $u_{d}=\left\{\phi_{d}, \theta_{d}, \delta_{c o l}\right\}$, such that $\varsigma \rightarrow \varsigma_{0}$ as $t \rightarrow \infty$ in the presence of external disturbance, where $\varsigma_{0}$ is the desired position. Now the translational dynamic of the helicopter system is given by

$$
\begin{aligned}
& \dot{\xi}=V \\
& m \dot{V}=R f_{b}+m g e_{3}+d
\end{aligned}
$$

where, $d$ is the external disturbance.

An original control strategy is proposed by combining an appropriate backstepping control with the use of a Lyapunov function allowing the attractive domain to be computed analytically

Step 1: The design process starts with the definition of the position tracking error and its derivative is as follows:

$$
\begin{aligned}
& z_{1}=\varsigma-\varsigma_{0} \\
& \dot{z}_{1}=V
\end{aligned}
$$

Here, $V$ is considered as a virtual control and $\mathrm{V} d$ is defined as the virtual control law in Eqn. (18). Let $z_{2}$ be an error variable representing the difference between the actual and virtual control of Eqn. (18) i.e., $z_{2}=V-V_{d}, V=z_{2}+V_{d}$. Therefore, $\dot{z}_{1}=z_{2}+V_{d}$

In this step the control objective is to design a virtual control law $V_{d}$ which would make $z_{l} \rightarrow 0$ as $t \rightarrow \infty$.

Now, consider a control Lyapunov function $W_{1}=\frac{1}{2} z_{1}{ }^{T} z_{1}$

And its derivative is, $\dot{W}_{1}=z_{1}^{T} \dot{z}_{1}, \quad \dot{W}_{1}=z_{1}^{T}\left(z_{2}+V_{d}\right)$

Now select an appropriate virtual control $V_{d}$ which would make $\dot{W}_{1} \leq 0$.

A possible choice is

$$
V_{d}=-\alpha_{p} z_{1}
$$

Then the time derivative of $W_{l}$ becomes

$$
\dot{W}_{1}=-\alpha_{p} z_{1}{ }^{T} z_{1}+z_{1}{ }^{T} z_{2}
$$

Clearly if $z_{2}=0$ then $\dot{W}_{1}=-\alpha_{p} z_{1}{ }^{T} z_{1} \leq 0$.

Now the time derivative of the (19) is as follows,

$$
\dot{\mathrm{V}}_{\mathrm{d}}=-\alpha_{\mathrm{p}} \dot{\mathrm{z}}_{1}, \dot{\mathrm{V}}_{\mathrm{d}}=-\alpha_{\mathrm{p}} \mathrm{V}
$$

Step 2: The error dynamic is derived for $z_{2}=V-V_{d}$ and its time derivative is given by,

$$
\begin{aligned}
& \dot{z}_{2}=\dot{V}-\dot{V}_{d}, \\
& \dot{z}_{2}=R \frac{f_{b}}{m}+g e_{3}+\frac{d}{m}+\alpha_{p} V
\end{aligned}
$$

Now a Control Lyapunov function (CLF) $W_{2}$ as follows,

$$
W_{2}=W_{1}+\frac{1}{2} z_{2}{ }^{T} z_{2}
$$

And its time derivative is, 


$$
\begin{aligned}
& \dot{W}_{2}=\dot{W}_{1}+z_{2}{ }^{T} \dot{z}_{2} \\
& \dot{W}_{2}=-\alpha_{p} z_{1}{ }^{T} z_{1}+z_{1}{ }^{T} z_{2}+z_{2}{ }^{T}\left(R \frac{f_{b}}{m}+g e_{3}+\frac{d}{m}+\alpha_{p} V\right)
\end{aligned}
$$

Backstepping algorithm stops at this step because control input $R f_{b}$ of translational dynamics appears.

Now

$$
\begin{aligned}
R f_{b}= & -m\left[z_{1}+\alpha_{p} V+\beta_{d} z_{2}+g e_{3}\right. \\
& \left.+\hat{\Delta}+F \operatorname{sgn}\left(z_{2}\right)\right]
\end{aligned}
$$

where, $\hat{\Delta}$ equal to an estimate of the unknown parameter $\Delta=\frac{d}{m}$. The estimation error on $\Delta$ is assumed to be bounded by knowing constant $F$, that is $|\Delta-\hat{\Delta}| \leq F$. Then the time derivative of $W_{2}$ becomes

$$
\dot{W}_{2}=-\alpha_{p} z_{1}{ }^{T} z_{1}-\beta_{d} z_{2}{ }^{T} z_{2}+z_{2}{ }^{T}\left(\Delta-\hat{\Delta}-F \operatorname{sgn}\left(z_{2}\right)\right)<0
$$

Where, the sign is Signum function and it is given as follows:

$$
\operatorname{sgn}\left(z_{2}\right)=\left\{\begin{array}{llc}
+1 & \text { if } & z_{2}>0 \\
0 & \text { if } & z_{2}=0 \\
-1 & \text { if } & z_{2}<0
\end{array}\right.
$$

Now the derivative of the final CLF is negative definite, the system will be stabilized at its equilibrium point.

\section{B. Inner-loop controller design}

The dynamics of rotational motion of a rigid-body helicopter under external wind gusts can be described by the following equations

$$
\begin{aligned}
& \dot{\eta}=\pi \omega \\
& I \dot{\omega}=-\omega \times I \omega+M+M_{\text {wind }}
\end{aligned}
$$

where, $M_{\text {wind }}$ represents the moment due to the external disturbance.

Step 1: The design process starts with the definition of the attitude tracking error i.e.,

$$
\tilde{\eta}=\eta-\eta_{d}
$$

Now, consider a control Lyapunov function (CLF)

$$
W_{1}=\frac{1}{2}\left(\eta-\eta_{d}\right)^{T} K_{\eta}\left(\eta-\eta_{d}\right)
$$

Where, $K_{\eta}$ is a positive definite matrix. The time derivative of $W_{l}$ is

$$
\begin{aligned}
\dot{W}_{1} & =\dot{\eta}^{T} K_{\eta} \tilde{\eta}, \\
& =(\pi \omega)^{T} K_{\eta} \tilde{\eta}
\end{aligned}
$$

If

$$
\omega=\omega_{d}=0 \quad \text { Then } \dot{W}_{1}=0 .
$$

The process of backstepping continues by defining error $e_{1}=\omega-\omega_{d}$ and having another control Lyapunov function as follows:

$$
W_{2}=W_{1}+\frac{1}{2} e_{1}^{T} I e_{1}
$$

And its time derivative is given by,

$$
\begin{aligned}
\dot{W}_{2} & =\dot{W}_{1}+e_{1}^{T} I \dot{e}_{1} \\
& =(\pi \omega)^{T} K_{\eta} \tilde{\eta}+e_{1}^{T}\left(-\omega \times I \omega+M+M_{\text {wind }}\right) \\
& =\pi^{T}\left(e_{1}+\omega_{d}\right)^{T} K_{\eta} \tilde{\eta}+e_{1}^{T}\left(-\omega \times I \omega+M+M_{\text {wind }}\right) \\
& =\omega_{d}{ }^{T} \pi^{T} K_{\eta} \tilde{\eta}+e_{1}^{T}\left(\pi^{T} K_{\eta} \tilde{\eta}-\omega \times I \omega+M+M_{\text {wind }}\right)
\end{aligned}
$$

Now

$$
M=\omega \times I \omega-\hat{M}_{\text {wind }}-\pi^{T} K_{\eta} \tilde{\eta}-F \operatorname{sgn}\left(e_{1}\right)
$$

where, $\hat{M}_{\text {wind }}$ equal to an estimation of the unknown parameter $M_{\text {wind }}$. The estimation error on $M_{\text {wind }}$ is assumed to be bounded by known constant $F$, that is $\left|M_{\text {wind }}-\hat{M}_{\text {wind }}\right| \leq F$. Then the time derivative of $W_{2}$ becomes

$$
\dot{W}_{2}=\omega_{d}{ }^{T} \pi^{T} K_{\eta} \tilde{\eta}+e_{1}^{T}\left(M_{\text {wind }}-\hat{M}_{\text {wind }}-F \operatorname{sgn}\left(e_{1}\right)\right)
$$

Hence, uesing Schwartzinquality, we have

$$
\begin{aligned}
\dot{W}_{2} & \leq \omega_{d}{ }^{T} \pi^{T} K_{\eta} \tilde{\eta}+\left|e_{1}\right|\left|M_{\text {wind }}-\hat{M}_{\text {wind }}\right|-F\left|e_{1}\right| \\
& \leq \omega_{d}{ }^{T} \pi^{T} K_{\eta} \tilde{\eta}-\left|e_{1}\right|\left[F-\left|M_{\text {wind }}-\hat{M}_{\text {wind }}\right|\right]
\end{aligned}
$$

Since, $\left|M_{\text {wind }}-\hat{M}_{\text {wind }}\right| \leq F$.

So,

$$
\dot{W}_{2} \leq 0 \text {. }
$$

Now the derivative of the final CLF is negative definite, the system will be stabilized at its equilibrium point.

\section{Simulation Result}

In this section, the simulation results are presented to investigate the performance of the proposed control algorithm to control of a Vario helicopter based on simulation parameters consistent with those employed in real applications. The control strategy is simulated for hovering flight at constant altitude. The helicopter initial and desired positions are set to $x=0 \mathrm{~m}, y=0 \mathrm{~m}$ and $z=-2 \mathrm{~m}$. The robust backstepping controller is designed to hold this position. The roll angle trim $\phi_{\text {ref }}$ is initialized at $4.5^{\circ}$ to compensate the tail rotor thrust. The heading of the Vario helicopter is initialized at $\psi_{\text {ref }}=0^{\circ}$ to point in the North direction. The 
dynamic model in Eq. (1)-(4) is implemented with the parameters listed in Table I. In order to verify the robustness of the proposed backstepping controller for position control of a hover flight, the bound on the external uncertainty is found $F=0.3 \mathrm{~m} / \mathrm{s}^{2}$ based on the accelerations and moment variation, respectively. The simulation result of the robust backstepping controller for position control is presented in Fig.1. The position of the Vario helicopter drifted approximately $0.2 \mathrm{~m}$ in $y$ direction up to $3 \mathrm{~s}$ from the beginning of the simulation and stayed approximately at -2 $\mathrm{m}$ above the ground. The drift error of the Vario helicopter in $x, y$, and $z$ direction shows that the helicopter can hover at the desired position in the presence of external wind gusts. The linear velocities are illustrated in Fig.2. It is clear that the linear velocities are settled to approximately $0 \mathrm{~m} / \mathrm{s}$ at about $3 \mathrm{~s}$ after the start of the simulation in $y$ and $z$ direction, respectively and $0 \mathrm{~m} / \mathrm{s}$ in $\mathrm{x}$ direction from the beginning of the simulation.

Table 1. Parameter of the vario helicopter

\begin{tabular}{|c|c|}
\hline Parameter & Description \\
\hline$a=5.7$ & Lift curve slope \\
\hline$b=3$ & Number of blades \\
\hline$m=27.738(\mathrm{Kg})$ & Vario helicopter mass \\
\hline$\Omega=850(\mathrm{rad} / \mathrm{sec})$ & Main rotor speed \\
\hline$\Omega_{t r}=4598.5(\mathrm{rad} / \mathrm{sec})$ & Tail rotor speed \\
\hline $\mathrm{R}=1.25(\mathrm{~m})$ & Main rotor radius \\
\hline$R_{t r}=0.42(\mathrm{~m})$ & Tail rotor radius \\
\hline$I_{x x}=12.3\left(\mathrm{Kg} \cdot \mathrm{m}^{2}\right)$ & Rolling moment of inertia \\
\hline$I_{y y}=18.7\left(\mathrm{Kg} \cdot \mathrm{m}^{2}\right)$ & Pitching moment of inertia \\
\hline$I_{z z}=6.6\left(\mathrm{Kg} \cdot \mathrm{m}^{2}\right)$ & Yawing moment of inertia \\
\hline$\rho=1.25\left(\mathrm{Kg} / \mathrm{m}^{3}\right)$ & Air density \\
\hline$M_{x}=0.03 \mathrm{~m}$ & Main rotor $\mathrm{x}$ coord w.r.t C.G \\
\hline$M_{y}=-0.0029 \mathrm{~m}$ & Main rotor y coord w.r.t C.G \\
\hline$M_{z}=-0.3321 \mathrm{~m}$ & Main rotor $\mathrm{z}$ coord w.r.t C.G \\
\hline$T_{x}=-1.44 \mathrm{~m}$ & Tail rotor $\mathrm{x}$ coord w.r.t C.G \\
\hline$T_{y}=-0.0029 \mathrm{~m}$ & Tail rotor y coord w.r.t C.G \\
\hline$T_{z}=1.1379 \mathrm{~m}$ & Tail rotor z coord w.r.t C.G \\
\hline$C_{m r}=0.076 \mathrm{~m}$ & Main rotor blade chord \\
\hline$C_{t r}=0.043 \mathrm{~m}$ & Tail rotor blade chord \\
\hline
\end{tabular}

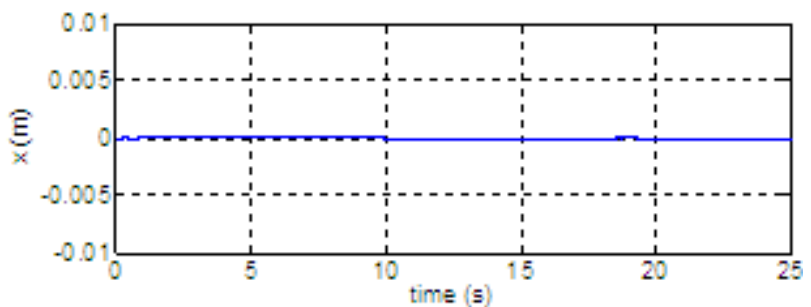

(a)

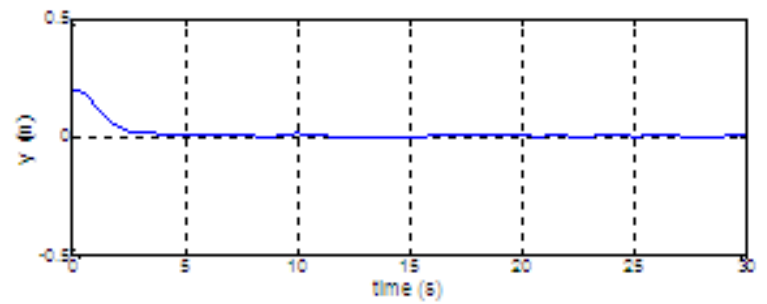

(b)

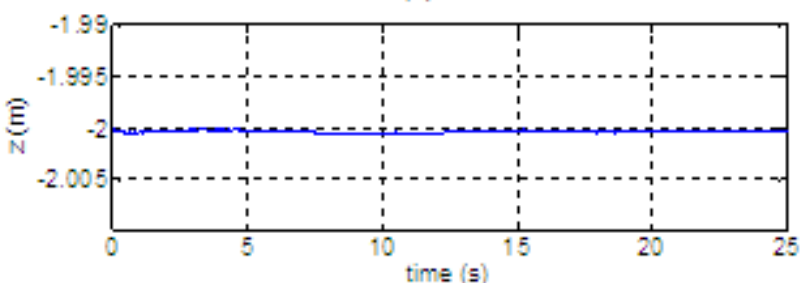

(c)

Figure 1. Vario helicopter position response in hover flight

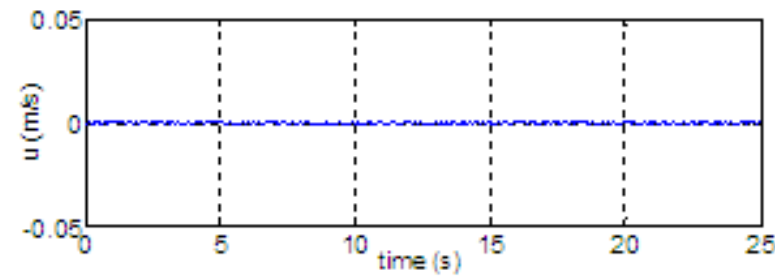

(a)

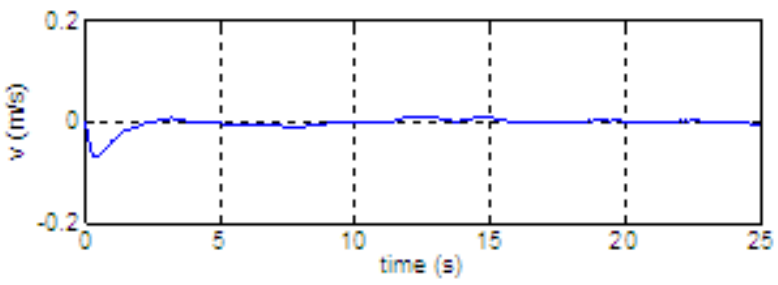

(b)

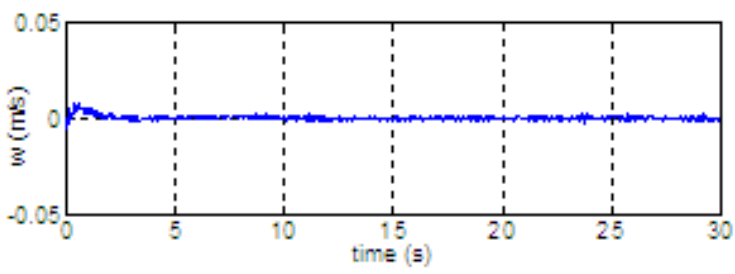

(c)

Figure 2. Vario helicopter linear velocities in hover flight 


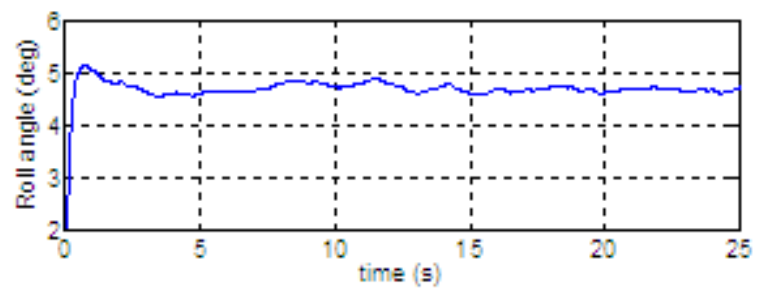

(a)

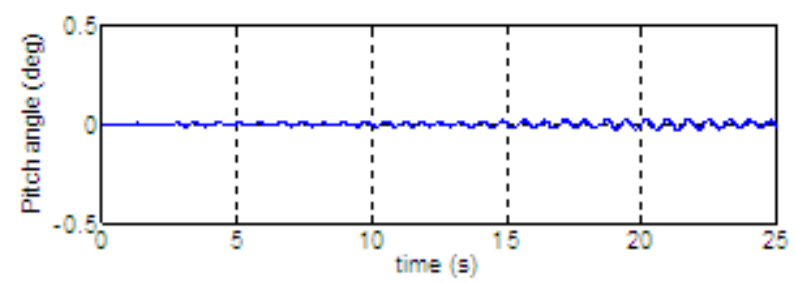

(b)

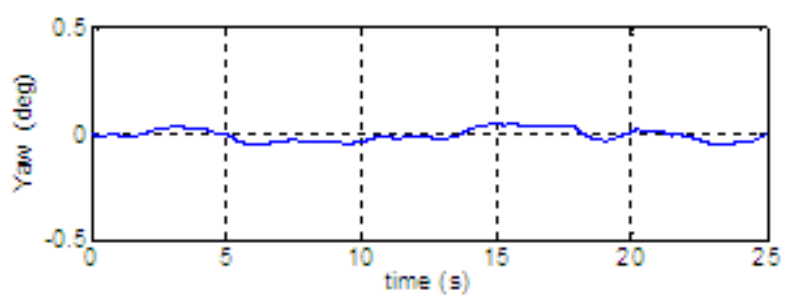

(c)

Figure3. Vario helicopter attitude response in hover flight

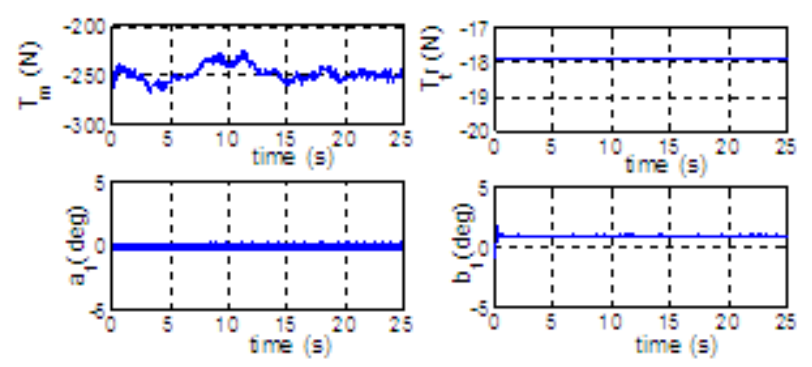

Figure 4. Behaviour of the control inputs

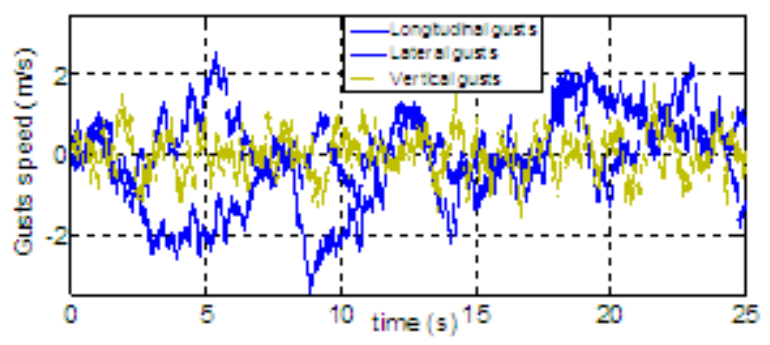

Figure 5. Wind gusts to test controller

Therefore, from the simulation results, it is obvious that the proposed controller is able to achieve the desired position along with altitude in the presence of external disturbances and stabilized the helicopter in hover flight. The attitude responses of the helicopter during hovering flight are shown Fig. 3 and it is clear that the angles converge to almost desired values. The control inputs obtained using robust backstepping are shown in Fig.4. They have not exceeded the constraints of the helicopter. The Dryden gust model shown in Fig.5 has been used in the simulation to test the controllers.

\section{Conclusion}

This paper has proposed an original approach, based on hierarchical structure (inner and outer loop) robust backstepping control law to track a hover flight trajectory with a 6-DOF nonlinear dynamics of miniature VARIO helicopter in the presence of non-constant wind gusts. Simulation results demonstrated that the proposed robust backstepping controller is able to attenuate the gusts effects in a hover flight condition and reached the desired position as well as attitude. This new method combines the advantages of both for the inner loop and for outer loop; particularly it will be simple and easy to implement and tune in the future real flight test. Future works will deal with the implementation of the proposed autonomous flight control method on the real system and flight test to prove its feasibility in real applications.

\section{REFERENCES}

[1] Xiaodong Wang and Xiaoguang Zhao, "A practical survey on the flight control system of small-scale unmanned helicopter", 7th World Congress on Intelligent Control and Automation (WCICA 2008), pp.364-369, 2008.

[2] Guowei Cai, Ben M. Chen, Xiangxu Dong, Tong H. Lee, Design and implementation of a robust and nonlinear flight control system for an unmanned helicopter, Mechatronics 21 (2011) 803-820.

[3] M. Chen, S.S. Ge, B. Ren, Robust attitude control of helicopter with actuator dynamics using neural networks, IET Control and Application, 2010, Vol. 4, Iss. 12, pp. 2837-2854.

[4] Dongkai SHEN, Jingjing WANG, Zhenghua LIU, Robust Backstepping Control based DRNN for Flight Simulator, Advanced Materials Research, Vols. 139-141 (2010) pp. 1708-1713.

[5] Bilal Ahmed and Hemanshu R. Poto, Flight Control of a Rotary Wing UAV using Adaptive Backstepping, IEEE International Conference on Control and Automation Christchurch, New Zealand, December 9-11, 2009.

[6] Kumeresan A. Danapalasingam, John-Josef Leth, Anders la Cour-Harbo and Morten Bisgaard, Robust Helicopter Stabilization in the Face of Wind Disturbance, 49th IEEE Conference on Decision and Control, December 15-17, 2010 Hilton Atlanta Hotel, Atlanta, GA, USA.

[7] T. Cheviron, F. Plestan, and A. Chriette, "A robust guidance and control scheme of an autonomous scale helicopter in presence of wind gusts," International Journal of Control, Vol. 82, No. 12 December 2009, 2206-2220. 
[8] Ciann-Dong Yang, Wen-Hsiung Liu and Chien-Chung Kung, "Robust Nonlinear $\mathrm{H}$ inf Decoupling Control of Flight Vehicle in Hovering", Proceedings of the 41sl IEEE Conference on Decision and Control ,Las Vega, Nevada USA, December 2002.

[9] Bilal Ahmed and Farid Kendoul, "Flight control of a small helicopter in unknown wind condition," 49th IEEE Conference on decision and Control, Hilton Atlanta Hotel, Atlanta, GA, USA, December 15-17, 2010.
[10] T. K. Roy, H. R. Pota, M. Garratt and H. Teimoori, "Robust Control for Longitudinal and Lateral Dynamics of Small Scale Helicopter," IEEE 31st Chinese Control Conference (CCC), 25-27 July 2012, Hefei, China, 2012 , pp. 2607-2612.

[11] Tushar Kanti Roy, "Robust backstepping control for small helicopter" Masters of Engineering thesis, November 2012, The University of New South Wales, Australia. 